\title{
NON-TAYLOR SERIES BASED POSITIONING METHOD FOR LOCATION BASED SERVICES
}

\author{
Tan Kim Geok, Ahmed Wasif Reza and San Choy Liew \\ Faculty of Engineering \& Technology \\ Multimedia University \\ Jalan Ayer Keroh Lama, Bukit Beruang \\ 75450 Melaka, Malaysia \\ awreza98@yahoo.com
}

\begin{abstract}
Location Based Services (LBS) has gained increasing popularity in major cities. Due to blocking from man-made structures, the existing Global Positioning System (GPS) could not satisfy LBS applications, especially in street canyon and indoor surroundings. This has lead to the development of Assisted GPS (A-GPS) which can provide better service availability and accuracy gain. In the conventional positioning method, Taylor series expansion is applied to solve non-linear distance equations. This method requires an initial estimation of A-GPS receiver's position. This paper investigates the positioning method for LBS based on hybrid E-OTD/GNSS. The proposed positioning method is non-Taylor series based. Therefore, it involves less complicated mathematical expansion and substitution. A flexible LBS positioning tool is developed which can generate position information in convenient way. It supports both Taylor series and non-Taylor series based positioning methods. The obtained results showed that the proposed non-Taylor series based positioning method can achieve better positioning accuracy.
\end{abstract}

Keywords: Location based services, Taylor series, Positioning system.

\section{INTRODUCTION}

The use of Global Positioning System (GPS) allows determination of position with high accuracy at anytime and anywhere. Therefore, GPS plays a vital role in aviation and maritime sectors. However, GPS has poor service availability 
in major cities especially in street canyons. This is because GPS signals are blocked by high-rise buildings and even tree canopies planted along streets. This has led to the development of a new concept of positioning known as Assisted Global Positioning System (A-GPS) or sometimes referred to as hybrid GPS.

A-GPS will play an important role in future as location based services (LBS) and has gained increasing popularity in major cities (Soliman \& Wheatley, 2002; Zhao, 2002). These applications require more stringent service availability and accuracy gain that could be only satisfied by A-GPS (MartinEscalona, Barcelo, \& Paradelis 2002; Zhao, 2000). A-GPS poses several advantages over conventional GPS, such as higher accuracy and availability gain, shorter time to first fix (TTFF), and works well even in indoor settings. A-GPS is more economical since it needs only one or more satellites in view. This is because it utilizes the existing mobile network to substitute partially the role of the satellite in positioning. Furthermore, most calculations involved in positioning is done on the mobile network side. This reduces the memory storage and processing power required by the A-GPS receiver (RX).

Position calculation for A-GPS can be solved by Taylor series based positioning method which applies a Taylor series expansion to solve nonlinear distance equations (Kaplan, 1996). It is also widely used in conventional GPS positioning. Its accuracy is influenced by the initial position estimation of RX. In this paper, non-Taylor series based positioning method is proposed for A-GPS. A detailed discussion on the conventional Taylor series based and non-Taylor series based positioning methods for hybrid GPS/Cellphone can be found in (Tan \& Tan, 2006). In contrast, this paper investigates the positioning method for LBS based on hybrid E-OTD/GNSS developed in the EMILY project (Martin-Escalona et al., 2002). The method proposed in this paper involves only simple equation expansion and substitution. This is a more efficient and simpler method. No initial estimation of RX position is required for this positioning method and it is able to generate more accurate positioning results as explained in the following sections. However, additional ranging information either from base station (BTS) or satellite (SV) is required for the proposed positioning method.

The remaining part of this paper is organised as follows. Firstly, this paper introduces the basics of positioning which covers earth-centered earth-fixed (ECEF) and geodetic coordinate systems. This is followed by a detailed equation derivation for Taylor series based and the proposed non-Taylor series based A-GPS positioning methods for hybrid E-OTD/GNSS. In the next section, a discussion on position calculation is presented and lastly, a comparison of positioning accuracy results is discussed. 


\section{BASICS OF POSITIONING}

GPS utilises the concept of time of arrival (TOA) of ranging signal (Farrell \& Barth, 1999) to determine RX position. With a single signal source, the surface of the sphere is the possible position. With two signal sources, the area of intersection of two spheres, which is a circle, is the possible position. With three signal sources, there are two points of possible solutions as shown in Figure 1. The point that is located on the earth is the expected position, while the other point which is located at the space is the ambiguity resulted from the interception and is the incorrect result to be omitted.

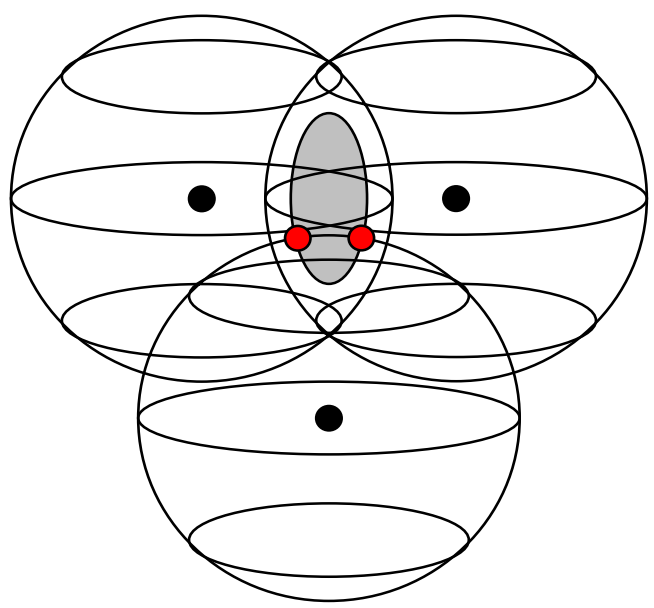

2 points of solution

Fig. 1: 3D Positioning

There are two important coordinate systems in global positioning which are earth-centered earth-fixed (ECEF) and geodetic (Kaplan, 1996) coordinate systems. The ECEF coordinate system is based on the Cartesian coordinate system of axis $x, y$, and $z$, while the geodetic coordinate system is based on Spherical coordinates which comprises longitude, latitude, and altitude. A positioning tool is developed in this work in order to generate positioning information based on Taylor series based and the proposed non-Taylor series based positioning methods. This tool allows users to enter positioning information in the geodetic coordinate system, while the internal position computation is based on ECEF coordinate system. The smooth sphere of earth surface is assumed with fixed radius, $r$ of $6371.3 \mathrm{~km}$ in this study. The ECEF coordinate $(x, y, z)$ can be converted from the geodetic coordinate $(\lambda, \phi, h)$, as shown in Figure 2 by applying the following equations. 


$$
x= \begin{cases}\sqrt{\frac{w^{2}}{1+\tan ^{2}(\lambda)}}, & -90 \leq \lambda<90 \\ -\sqrt{\frac{w^{2}}{1+\tan ^{2}(\lambda)}}, & 90 \leq \lambda<180 \text { or }-180 \leq \lambda<-90\end{cases}
$$

$$
y=\left\{\begin{aligned}
\sqrt{w^{2}-x^{2}}, & 0 \leq \lambda<180 \\
-\sqrt{w^{2}-x^{2}}, & -180 \leq \lambda<0
\end{aligned}\right.
$$

$$
z=\left\{\begin{array}{cc}
\sqrt{d^{2}-w^{2}}, & 0 \leq \phi<90 \\
-\sqrt{d^{2}-w^{2}}, & -90 \leq \phi<0
\end{array}\right.
$$



Fig. 2: Coordinates Conversion from Geodetic Coordinates $(\lambda, \phi, h)$ to ECEF Coordinate $(x, y, z)$.

\section{TAYLOR SERIES BASED POSITIONING METHOD}

The enhanced observe time difference (OTD) of hybrid E-OTD/GNSS (Martin-Escalona et al., 2002) can be obtained by differencing distance equation $\bar{P}_{i}$ and $\bar{P}_{j}$, where $\bar{P}_{i}=\sqrt{\left(x_{i}-x\right)^{2}+\left(y_{i}-y\right)^{2}+\left(z_{i}-z\right)^{2}}+c t_{c}$. Note that the RX clock offset, $t_{c}$, is cancelled off during differencing, and $c$ is speed of light. 
In the Taylor series based positioning method, initial RX position estimation is required. More details are available in Farrell and Barth (1999), Kaplan (1996), Krasner, Marshall, and Riley (2002), and Soliman, Agashe, Fernandez, Vayanos, Goal, \& Oljaca (2000). The OTD, $d_{i j}$ is written in general form with reference to station $i$ and station $j$ as below.

$$
\begin{aligned}
\bar{P}_{i}-\bar{P}_{j}=d_{i j} & =\sqrt{\left(x_{i}-x\right)^{2}+\left(y_{i}-y\right)^{2}+\left(z_{i}-z\right)^{2}} \\
& -\sqrt{\left(x_{j}-x\right)^{2}+\left(y_{j}-y\right)^{2}+\left(z_{j}-z\right)^{2}} \\
& =f(x, y, z)
\end{aligned}
$$

By substituting RX position with the estimated values, an approximate function is obtained in the following.

$$
\begin{aligned}
\hat{d}_{i j}= & \sqrt{\left(x_{i}-\hat{x}\right)^{2}+\left(y_{i}-\hat{y}\right)^{2}+\left(z_{i}-\hat{z}\right)^{2}} \\
& -\sqrt{\left(x_{j}-\hat{x}\right)^{2}+\left(y_{j}-\hat{y}\right)^{2}+\left(z_{j}-\hat{z}\right)^{2}} \\
= & f(\hat{x}, \hat{y}, \hat{z})
\end{aligned}
$$

The unknown RX position can be written as its approximated value plus the position offset; $x=\hat{x}+\Delta x, y=\hat{y}+\Delta y$, and $z=\hat{z}+\Delta z$. Thus, the function can be rewritten as:

$$
f(x, y, z)=f(\hat{x}+\Delta x, \hat{y}+\Delta y, \hat{z}+\Delta z)
$$

This is followed by expanding the final function by using Taylor series expansion as shown in Equation (7).

$$
\begin{aligned}
& f(\hat{x}+\Delta x, \hat{y}+\Delta y, \hat{z}+\Delta z)=f(\hat{x}, \hat{y}, \hat{z})+\frac{\partial f(\hat{x}, \hat{y}, \hat{z})}{\partial \hat{x}} \Delta x \\
& +\frac{\partial f(\hat{x}, \hat{y}, \hat{z})}{\partial \hat{y}} \Delta y+\frac{\partial f(\hat{x}, \hat{y}, \hat{z})}{\partial \hat{z}} \Delta z+\ldots
\end{aligned}
$$

Let us consider for the first-order partial derivatives to eliminate the nonlinear terms. The partial derivatives are shown as follows. 


$$
\begin{aligned}
\frac{\partial f(\hat{x}, \hat{y}, \hat{z})}{\partial \hat{x}}= & \frac{x_{j}-\hat{x}}{\sqrt{\left(x_{j}-\hat{x}\right)^{2}+\left(y_{j}-\hat{y}\right)^{2}+\left(z_{j}-\hat{z}\right)^{2}}} \\
& -\frac{x_{i}-\hat{x}}{\sqrt{\left(x_{i}-\hat{x}\right)^{2}+\left(y_{i}-\hat{y}\right)^{2}+\left(z_{i}-\hat{z}\right)^{2}}} \\
\frac{\partial f(\hat{x}, \hat{y}, \hat{z})}{\partial \hat{y}}= & \frac{y_{j}-\hat{y}}{\sqrt{\left(x_{j}-\hat{x}\right)^{2}+\left(y_{j}-\hat{y}\right)^{2}+\left(z_{j}-\hat{z}\right)^{2}}} \\
\frac{\partial f(\hat{x}, \hat{y}, \hat{z})}{\partial \hat{z}}= & -\frac{y_{i}-\hat{y}}{\sqrt{\left(x_{i}-\hat{x}\right)^{2}+\left(y_{i}-\hat{y}\right)^{2}+\left(z_{i}-\hat{z}\right)^{2}}} \\
& -\frac{z_{j}-\hat{z}}{\sqrt{\left(x_{j}-\hat{x}\right)^{2}+\left(y_{j}-\hat{y}\right)^{2}+\left(z_{j}-\hat{z}\right)^{2}}}
\end{aligned}
$$

Let, the denominator parts of each of the partial derivative resultant is represented by $\hat{r}_{i}=\sqrt{\left(x_{i}-\hat{x}\right)^{2}+\left(y_{i}-\hat{y}\right)^{2}+\left(z_{i}-\hat{z}\right)^{2}}$ and $\hat{r}_{j}=\sqrt{\left(x_{j}-\hat{x}\right)^{2}+\left(y_{j}-\hat{y}\right)^{2}+\left(z_{j}-\hat{z}\right)^{2}}$.

Substituting Equations (7) and (8) into Equation (4), a new equation is obtained as in the following:

$$
\begin{aligned}
d_{i j}= & \hat{d}_{i j}+\left(\frac{x_{j}-\hat{x}}{\hat{r}_{j}}-\frac{x_{i}-\hat{x}}{\hat{r}_{i}}\right) \Delta x+\left(\frac{y_{j}-\hat{y}}{\hat{r}_{j}}-\frac{y_{i}-\hat{y}}{\hat{r}_{i}}\right) \Delta y \\
& +\left(\frac{z_{j}-\hat{z}}{\hat{r}_{j}}-\frac{z_{i}-\hat{z}}{\hat{r}_{i}}\right) \Delta z
\end{aligned}
$$

The above equation is simplified by introducing the new variables as below. 


$$
\begin{aligned}
& \Delta d_{i j}=\hat{d}_{i j}-d_{i j} \\
& a_{x i j}=\frac{x_{i}-\hat{x}}{\hat{r}_{i}}-\frac{x_{j}-\hat{x}}{\hat{r}_{j}}=\frac{\hat{r}_{j}\left(x_{i}-\hat{x}\right)-\hat{r}_{i}\left(x_{j}-\hat{x}\right)}{\hat{r}_{i} \hat{r}_{j}} \\
& a_{y i j}=\frac{y_{i}-\hat{y}}{\hat{r}_{i}}-\frac{y_{j}-\hat{y}}{\hat{r}_{j}}=\frac{\hat{r}_{j}\left(y_{i}-\hat{y}\right)-\hat{r}_{i}\left(y_{j}-\hat{y}\right)}{\hat{r}_{i} \hat{r}_{j}} \\
& a_{z i j}=\frac{z_{i}-\hat{z}}{\hat{r}_{i}}-\frac{z_{j}-\hat{z}}{\hat{r}_{j}}=\frac{\hat{r}_{j}\left(z_{i}-\hat{z}\right)-\hat{r}_{i}\left(z_{j}-\hat{z}\right)}{\hat{r}_{i} \hat{r}_{j}}
\end{aligned}
$$

Thus, a new linear equation is obtained as follows.

$$
\Delta d_{i j}=a_{x i j} \Delta x+a_{y i j} \Delta y+a_{z i j} \Delta z
$$

This equation has three unknowns to be solved. This equation is expanded by making time difference measurements to obtain a set of three linear equations which can easily be solved in matrix form as presented below.

$$
\Delta d=\left[\begin{array}{l}
\Delta d_{12} \\
\Delta d_{23} \\
\Delta d_{34}
\end{array}\right] \quad H=\left[\begin{array}{lll}
a_{x 12} & a_{y 12} & a_{z 12} \\
a_{x 23} & a_{y 23} & a_{z 23} \\
a_{x 34} & a_{y 34} & a_{z 34}
\end{array}\right] \quad \Delta X=\left[\begin{array}{c}
\Delta x \\
\Delta y \\
\Delta z
\end{array}\right]
$$

Finally, the displacement can be obtained as $\Delta X=H^{-1} \Delta d$. Therefore, the final RX position can be obtained as $x=\hat{x}+\Delta x, y=\hat{y}+\Delta y$, and $z=\hat{z}+\Delta z$

\section{NON-TAYLOR SERIES BASED POSITIONING METHOD}

The discussion of the proposed non-Taylor series based positioning method for hybrid E-OTD/GNSS is started by rewriting Equation (4) as below.

$$
\begin{aligned}
& d_{i j}{ }^{2}+2 d_{i j} \sqrt{\left(x_{j}-x\right)^{2}+\left(y_{j}-y\right)^{2}+\left(z_{j}-z\right)^{2}} \\
& +\left[\left(x_{j}-x\right)^{2}+\left(y_{j}-y\right)^{2}+\left(z_{j}-z\right)^{2}\right] \\
& =\left(x_{i}-x\right)^{2}+\left(y_{i}-y\right)^{2}+\left(z_{i}-z\right)^{2}
\end{aligned}
$$


For further expansion, substituting $p_{j}=\sqrt{\left(x_{j}-x\right)^{2}+\left(y_{j}-y\right)^{2}+\left(z_{j}-z\right)^{2}}$ into Equation (13), Equation (14) is obtained in the following.

$$
\begin{aligned}
& p_{j}=\frac{\left(x_{i}^{2}+y_{i}{ }^{2}+z_{i}^{2}\right)-\left(x_{j}{ }^{2}+y_{j}{ }^{2}+z_{j}{ }^{2}\right) \cdots}{2 d_{i j}} \\
& \frac{+2\left(x_{j}-x_{i}\right) x+2\left(y_{j}-y_{i}\right) y+2\left(z_{j}-z_{i}\right) z-d_{i j}{ }^{2}}{2 d_{i j}}
\end{aligned}
$$

The above equation is simplified by introducing the new variables; $a_{i j}=\left(x_{i}{ }^{2}+y_{i}{ }^{2}+z_{i}{ }^{2}\right)-\left(x_{j}{ }^{2}+y_{j}{ }^{2}+z_{j}{ }^{2}\right)-d_{i j}{ }^{2}, a_{x i j}=2\left(x_{j}-x_{i}\right)$, $a_{y i j}=2\left(y_{j}-y_{i}\right)$, and $a_{z i j}=2\left(z_{j}-z_{i}\right)$. Thus, a new equation is obtained as follows.

$$
p_{j}=\frac{a_{i j}+a_{x i j} x+a_{y i j} y+a_{z i j} z}{2 d_{i j}}
$$

Equation (15) is expanded based on time difference measurements to get a set of four non-linear equations as below (in this case $j=1$, since all the E-OTD measurements are made with respect to station 1, i.e. $d_{21}, d_{31}, d_{41}$, and $d_{51}$ ).

$$
\begin{aligned}
& p_{1}=\frac{a_{21}+a_{x 21} x+a_{y 21} y+a_{z 21} z}{2 d_{21}} \\
& p_{1}=\frac{a_{31}+a_{x 31} x+a_{y 31} y+a_{z 31} z}{2 d_{31}} \\
& p_{1}=\frac{a_{41}+a_{x 41} x+a_{y 41} y+a_{z 41} z}{2 d_{41}} \\
& p_{1}=\frac{a_{51}+a_{x 51} x+a_{y 51} y+a_{z 51} z}{2 d_{51}}
\end{aligned}
$$

Now, the above equations are solved by subtracting (16a) with (16b), (16b) with (16c), and (16c) with (16d). A new set of equations are obtained and can be represented by a single general equation as below. 


$$
\begin{aligned}
& 2\left(d_{k} a_{l}-d_{l} a_{k}\right)=2\left(d_{l} a_{x k}-d_{k} a_{x l}\right) x \\
& +2\left(d_{l} a_{y k}-d_{k} a_{y l}\right) y+2\left(d_{l} a_{z k}-d_{k} a_{z l}\right) z
\end{aligned}
$$

Equation (17) is further simplified by introducing new variables $b_{k l}=2\left(d_{k} a_{l}-d_{l} a_{k}\right), b_{x k l}=2\left(d_{l} a_{x k}-d_{k} a_{x l}\right), b_{y k l}=2\left(d_{l} a_{y k}-d_{k} a_{y l}\right)$, and $b_{z k l}=2\left(d_{l} a_{z k}-d_{k} a_{z l}\right)$. Thus, a new equation is obtained as shown below.

$$
b_{k l}=b_{x k l} x+b_{y k l} y+b_{z k l} z
$$

Equation (18) is expanded to get a set of three linear equations, which can easily be solved in matrix form as below.

$$
a=\left[\begin{array}{l}
b_{23} \\
b_{34} \\
b_{45}
\end{array}\right] \quad H=\left[\begin{array}{lll}
b_{x 23} & b_{y 23} & b_{z 23} \\
b_{x 34} & b_{y 34} & b_{z 34} \\
b_{x 45} & b_{y 45} & b_{z 45}
\end{array}\right] \quad k=\left[\begin{array}{c}
x \\
y \\
z
\end{array}\right]
$$

Lastly, the final RX position can be solved as $k=H^{-1} a$.

\section{RESULTS}

In this study, a LBS position computation tool was developed to implement the Taylor series and the proposed non-Taylor series based positioning methods. When the tool is executed, the RX starts searching for ranging signals. Then, the tool based on flowchart shown in Figure 3 decides the positioning method to be used depending on the number of detected ranging signals. It is also possible to predetermine the preferred positioning methods provided the required ranging signals are fulfilled. The tool allows flexible setting of position for satellites and base stations, the level of noise, and delays. The following results are generated using this tool. 


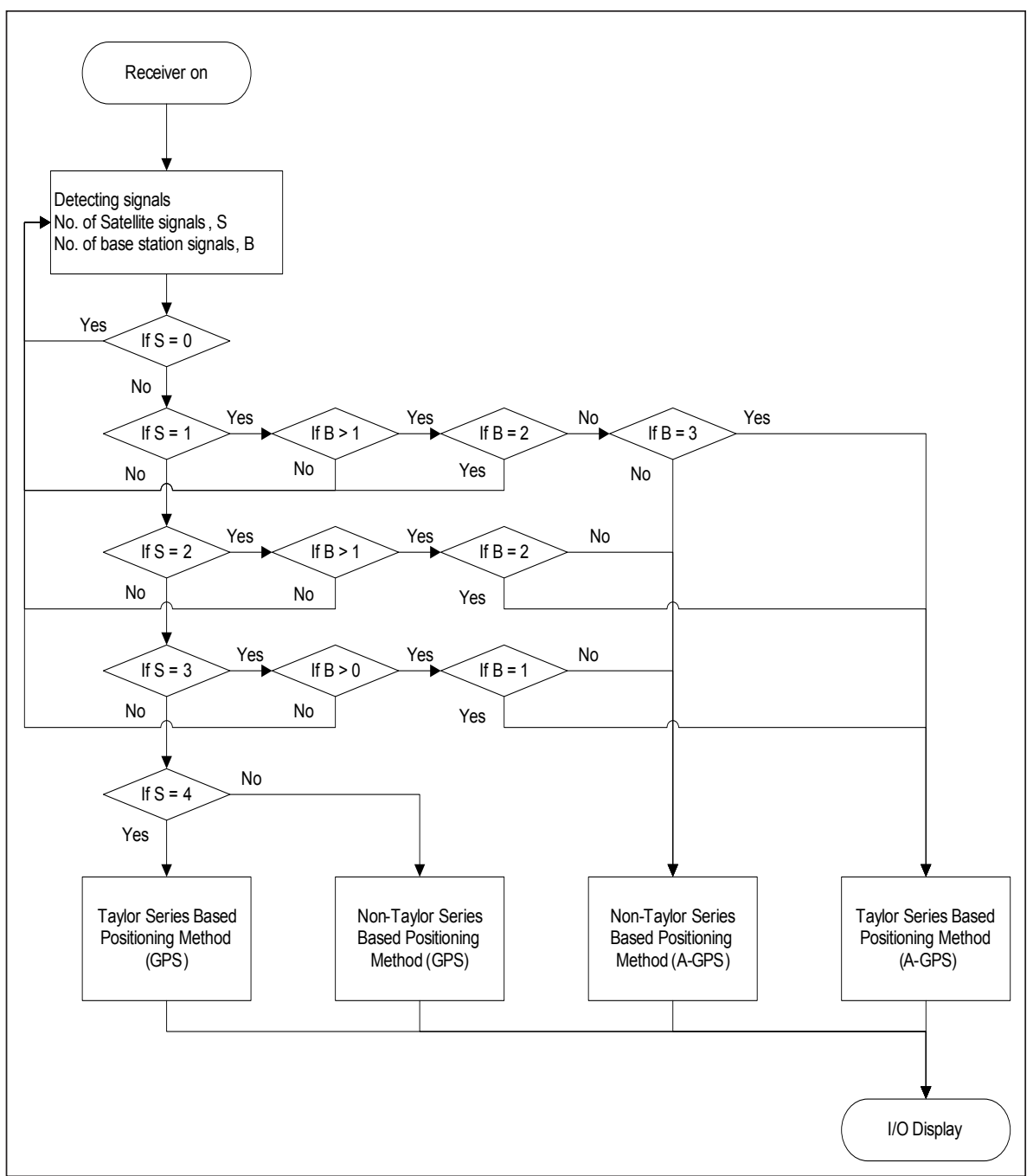

Fig. 3: Positioning Method Selection of A-GPS Receiver Based on Availability of Sufficient Ranging Signals

All SVs are located about $20,200 \mathrm{~km}$ from the earth surface. This is equivalent to satellite's orbital radius of about $26,600 \mathrm{~km}$. Table 1 summarises the five different sets of Geodetic coordinates for SVs, BTSs, and RXs which are used by all position calculations in this paper. The positioning accuracy is measured from the difference between the calculated position and the actual position of $\mathrm{RX}$ as expressed in Equation (20). In this paper, the initial estimation of RX position is identical to actual $\mathrm{RX}$ position in order to achieve a fair comparison between different positioning methods. 
accuracy $=\sqrt{\left(x_{c a l}-x_{a c t}\right)^{2}+\left(y_{c a l}-y_{a c t}\right)^{2}+\left(z_{c a l}-z_{a c t}\right)^{2}}$

Positioning accuracy can be calculated in terms of horizontal and vertical accuracy, as shown below.

accuracy $_{\text {hor }}=\sqrt{\left(\text { longitude }_{\text {cal }}-\text { longitude }_{\text {act }}\right)^{2}+\left(\text { latitude }_{\text {cal }}-\text { latitude }_{\text {act }}\right)^{2}}$

accuracy $_{\text {ver }}=\mid$ altitude $_{\text {cal }}-$ altitude $_{\text {act }} \mid$

where hor and ver represents the horizontal and vertical, respectively. Note that the horizontal accuracy is measured in terms of degree. Thus, it will be required to convert it into metre or kilometre. The conversion can be done as follows with an assumption that the Earth is an exact sphere, with a mean radius, $r$ of $6371.3 \mathrm{~km}$ (as mentioned before), where circumference $=2 \times \pi \times$ $r=40032 \mathrm{~km}$.

accuracy $_{\text {hor }, k m}=\frac{\text { accuracy }_{\text {hor }} \times \text { circumference }}{360}$

Results discussion of hybrid E-OTD/GNSS can be divided into two parts. The first part discusses accuracy comparison of position calculation between Taylor series based and the proposed non-Taylor series based positioning methods with the absence of delays and noises (Drakoulis, Kyria, \& Karetsos 2000; Narenthiran, Tafazolli, \& Evans, 2000; Van Diggelen \& Brown, 1994).

Table 2 summarises the calculated positioning accuracy in the absence of noises and delays for all geodetic coordinates as shown in Table 1. The obtained results showed that both the Taylor series based and non-Taylor series based positioning methods can achieve high positioning accuracy in the absence of delay and noise, where the calculated RX positions are close to the actual RX positions. However, an overall comparison showed that the proposed non-Taylor series based positioning method can achieve higher positioning accuracy as compared to Taylor series based positioning method. 
Table 1: Five Different Sets of Geodetic Coordinates for SVs, BTSs, and RXs, and Positioning Accuracy Results without the Presence of Noises and Delays

\begin{tabular}{|c|c|c|c|c|c|c|c|}
\hline \multirow[b]{2}{*}{ Coordinates } & \multicolumn{4}{|c|}{ SV and BTSs Coordinates } & \multicolumn{3}{|c|}{ RXs Coordinates } \\
\hline & $\begin{array}{l}\text { SVs/ } \\
\text { BTSs }\end{array}$ & Long $\left({ }^{\circ}\right)$ & Lat $\left({ }^{\circ}\right)$ & $\begin{array}{l}\text { Height } \\
(\mathrm{km})\end{array}$ & Long $\left(^{\circ}\right)$ & Lat $\left({ }^{\circ}\right)$ & $\begin{array}{l}\text { Height } \\
(\mathrm{km})\end{array}$ \\
\hline \multirow{5}{*}{ Set 1} & Satellite 2 & 81.7600 & -73.1783 & - & \multirow{5}{*}{102.9200} & \multirow{5}{*}{-56.0653} & \multirow{5}{*}{0.8160} \\
\hline & Satellite 3 & 86.7600 & 19.6543 & - & & & \\
\hline & Satellite 4 & 96.7600 & -51.7531 & - & & & \\
\hline & Satellite 5 & 96.7600 & 33.9358 & - & & & \\
\hline & BTS 1 & 102.9200 & -56.0652 & 1 & & & \\
\hline \multirow{5}{*}{ Set 2} & Satellite 1 & 96.6400 & 30.9081 & - & \multirow{5}{*}{104.5400} & \multirow{5}{*}{-39.1046} & \multirow{5}{*}{1.0920} \\
\hline & Satellite 2 & 104.6400 & -40.9493 & - & & & \\
\hline & Satellite 3 & 109.6400 & 52.3303 & - & & & \\
\hline & BTS 1 & 104.5400 & -39.1045 & 1 & & & \\
\hline & BTS 2 & 104.5400 & -39.1047 & 1.5 & & & \\
\hline \multirow{5}{*}{ Set 3} & Satellite 1 & 85.4000 & 17.7120 & - & \multirow{5}{*}{95.6000} & \multirow{5}{*}{7.5428} & \multirow{5}{*}{1.0000} \\
\hline & Satellite 2 & 95.4000 & -53.6954 & - & & & \\
\hline & Satellite 3 & 100.4000 & 39.1342 & - & & & \\
\hline & BTS 1 & 95.6001 & 95.6000 & 1 & & & \\
\hline & BTS 2 & 7.4286 & 7.4266 & 0.5 & & & \\
\hline \multirow{5}{*}{ Set 4} & Satellite 1 & 95.2400 & 31.7650 & - & \multirow{5}{*}{99.4400} & \multirow{5}{*}{38.9711} & \multirow{5}{*}{1.0000} \\
\hline & Satellite 2 & 105.2400 & 39.6424 & - & & & \\
\hline & BTS 1 & 99.4401 & 38.9312 & 1 & & & \\
\hline & BTS 2 & 99.4400 & 38.971 & 0.5 & & & \\
\hline & BTS 3 & 99.4398 & 38.971 & 1.3 & & & \\
\hline \multirow{5}{*}{ Set 5} & Satellite 1 & 107.1800 & 48.8171 & - & \multirow{5}{*}{101.0000} & \multirow{5}{*}{58.5659} & \multirow{5}{*}{0.8000} \\
\hline & Satellite 4 & 82.1800 & -72.5755 & - & & & \\
\hline & Satellite 5 & 82.1800 & 13.1134 & - & & & \\
\hline & BTS 1 & 101.0000 & 58.5660 & 1 & & & \\
\hline & BTS 2 & 101.0000 & 58.5658 & 0.5 & & & \\
\hline
\end{tabular}

Table 2: Calculated Positioning Accuracy Results for Hybrid E-OTD/ GNSS in the Absence of Noises and Delays

\begin{tabular}{ccc}
\hline \multirow{2}{*}{ Coordinates } & \multicolumn{2}{c}{ Accuracy $(\mathrm{km})$} \\
\cline { 2 - 3 } & Taylor Series & Non-Taylor Series \\
\hline Set 1 & $1.3860 \times 10^{-6}$ & $2.7004 \times 10^{-11}$ \\
Set 2 & $8.0992 \times 10^{-5}$ & $4.3970 \times 10^{-9}$ \\
Set 3 & $1.1359 \times 10^{-7}$ & $2.1077 \times 10^{-11}$ \\
Set 4 & $1.0406 \times 10^{-6}$ & $2.6561 \times 10^{-10}$ \\
Set 5 & $5.3993 \times 10^{-6}$ & $7.8345 \times 10^{-9}$ \\
\hline
\end{tabular}


In the second part of position calculation and accuracy comparison, delays and errors from different signal sources, such as receiver clock offset, atmospheric, ephemeris, multipath, and selective availability (SA) noise, were included in the computation. Receiver clock offset is identical for all signals, which is $5 \times 10^{-10}$ seconds $(\mathrm{sec})$. For BTS, atmospheric delay, ephemeris delay, and SA noise are irrelevant. Table 3 shows the delays and noise introduced by different signal sources. Let the sum of all the delays be represented by $\varepsilon$. The new time of travel is no longer the actual time of travel, but it is equal to the sum of actual time of travel and $\varepsilon$ as expressed in Equation (23)

$$
\mathrm{TOT}_{\mathrm{NEW}}=\mathrm{TOT}_{\mathrm{ACTUAL}}+\varepsilon
$$

Table 3: Delays and Noises

\begin{tabular}{lccccc}
\hline Signal Source & $\begin{array}{c}\text { Atmospheric } \\
(\mathrm{sec})\end{array}$ & $\begin{array}{c}\text { Ephemeris } \\
(\mathrm{sec})\end{array}$ & $\begin{array}{c}\text { Multipath } \\
(\mathrm{sec})\end{array}$ & $\begin{array}{c}\text { Noise } \\
(\mathrm{sec})\end{array}$ & $\begin{array}{c}\text { SA } \\
(\mathrm{sec})\end{array}$ \\
\hline Satellite 1 & $2.00 \times 10^{-8}$ & $1.70 \times 10^{-9}$ & $3.30 \times 10^{-10}$ & $3.30 \times 10^{-9}$ & $2.30 \times 10^{-8}$ \\
Satellite 2 & $1.95 \times 10^{-8}$ & $1.71 \times 10^{-9}$ & $3.31 \times 10^{-10}$ & $3.31 \times 10^{-9}$ & $2.35 \times 10^{-8}$ \\
Satellite 3 & $2.02 \times 10^{-8}$ & $1.69 \times 10^{-9}$ & $3.29 \times 10^{-10}$ & $3.29 \times 10^{-9}$ & $2.33 \times 10^{-8}$ \\
Satellite 4 & $2.03 \times 10^{-8}$ & $1.66 \times 10^{-9}$ & $3.35 \times 10^{-10}$ & $3.35 \times 10^{-9}$ & $2.29 \times 10^{-8}$ \\
Satellite 5 & $1.98 \times 10^{-8}$ & $1.74 \times 10^{-9}$ & $3.27 \times 10^{-10}$ & $3.27 \times 10^{-9}$ & $2.31 \times 10^{-8}$ \\
Base Station 1 & - & - & $4.00 \times 10^{-10}$ & $4.50 \times 10^{-9}$ & - \\
Base Station 2 & - & - & $4.05 \times 10^{-10}$ & $4.55 \times 10^{-9}$ & - \\
Base Station 3 & - & - & $3.99 \times 10^{-10}$ & $4.48 \times 10^{-9}$ & - \\
Base Station 4 & - & - & $4.01 \times 10^{-10}$ & $4.52 \times 10^{-9}$ & - \\
\hline
\end{tabular}

In the presence of noises and delays, the accuracy comparison further showed that the proposed non-Taylor series based positioning method also gave better results than the results obtained by using the Taylor series based positioning method. The Taylor series based positioning method achieved positioning accuracy in the range of 13 metre to 85 metre, as shown in Table 4, while the proposed method is giving positioning accuracy as low as less than 1 metre to 36 metre. The obtained better accuracy of non-Taylor series based positioning method is due to the additional ranging information involved in the position computation.

Table 4: Calculated Positioning Accuracy for Hybrid E-OTD/GNSS in the Presence of Noises and Delays

\begin{tabular}{ccc}
\hline \multirow{2}{*}{ Coordinates } & \multicolumn{2}{c}{ Accuracy $(\mathrm{km})$} \\
\cline { 2 - 3 } & Taylor Series & Non-Taylor Series \\
\hline Set 1 & 0.0238425 & 0.0007592 \\
Set 2 & 0.0199314 & 0.0157607 \\
Set 3 & 0.0130558 & 0.0129520 \\
Set 4 & 0.0171109 & 0.0113467 \\
Set 5 & 0.0857510 & 0.0367768 \\
\hline
\end{tabular}


Further analysis reveals that, the selection of BTS has significant influence on the positioning accuracy. It can be noted that, as the BTS gets closer to each other and/or closer to RX, the positioning accuracy is expected to decrease, because the time of travel of these signals become too short for the position computation. Based on the obtained results, the preferable distance between BTS and RX should be above $1 \mathrm{~km}$ in order to produce satisfactory accuracy results.

\section{CONCLUSION}

This paper has discussed in detailed the conventional Taylor series based and the proposed non-Taylor series based positioning methods for both hybrid E-OTD/GNSS and hybrid GPS/Cellphone systems. The proposed non-Taylor series based positioning method is a simpler and more accurate positioning method which involves less complicated mathematical substitution and expansion. The LBS position computation tool was developed to implement the above discussed positioning methods. The tool allows flexible setting of position for satellites and base stations, the level of noise and delays. The obtained results showed that the calculated or estimated RX position by the proposed method is very close to the actual RX position with the absence of delays and noises; thus, it achieves improvement on accuracy provided by the conventional Taylor series based positioning method. In contrast, with the presence of delays and noises, the proposed positioning method achieves positioning accuracy ranging from below 1 metre to 36 metres, which is also higher that the results obtained by using Taylor series based positioning method. A better positioning accuracy of the proposed non-Taylor series based positioning method can be achieved by including additional ranging information, which is available from the surrounding BTS. The other advantage of the proposed positioning method is that, no initial estimation of $\mathrm{RX}$ position is required. In a practical situation, the positioning accuracy of Taylor series based positioning method is influenced by the accuracy of the initial estimation of RX position.

\section{REFERENCES}

Drakoulis, D., Kyriazakos, S., \& Karetsos, G. (2000). Improving subscriber position location using a hybrid satellite-assisted and network-based technique. Vehicular Technology Conference, 4, 1887-1893.

Farrell, J. A., \& Barth, M. (1999). The global positioning system and inertial navigation. McGrawHill. 
Kaplan, E. D. (1996). Understanding GPS principles and applications. Norwood MA: Artech House.

Krasner, N. F., Marshall, G., \& Riley, W. (2002). Position determination using hybrid GPS/Cellphone ranging. Snap Track Division, a Qualcomm Company whitepaper.

Liew, S. C., Tan, K. G., \& Tan C. P. (2006). Non-Taylor series based positioning method for hybrid GPS/Cellphone system. Journal of Electromagnetic Waves and Applications, 20(6), 717-729.

Martin-Escalona, I., Barcelo, F., \& Paradelis, J. (2002, September). Delivery of non-standardised assistance data in E-OTD/GNSS hybrid location systems. Proceedings of the $13^{\text {th }}$ IEEE International Symposium on Personal, Indoor and Mobile Radio Communications: Vol. 5. (pp. 2347-2351). Lisbon.

Narenthiran, K., Tafazolli, R., \& Evans, B. G. (2000). Evaluation of location tracking schemes for satellite UMTS. First International Conference on $3 G$ Mobile Communication Technologies (pp. 381-386). London, UK.

Soliman, S., Agashe, P., Fernandez, I., Vayanos, A., Gaal, P., \& Oljaca, M. (2000). gpsONE ${ }^{\mathrm{TM}}$. A hybrid position location system. IEEE Sixth International Symposium on Spread Spectrum Techniques and Applications: Vol. 1. (pp. 330-335). New Jersey.

Soliman, S. S., \& Wheatley, C. E. (2002). Geolocation technologies and applications for third generation wireless. Wireless Communications and Mobile Computing, 2, 229-251.

Van Diggelen, F., \& Brown, A. (1994). Mathematical aspects of GPS RAIM. IEEE Position and Navigation Symposium (pp. 733-738). Las Vegas, $\mathrm{NV}$, USA.

Zhao, Y. (2000, March). Mobile phone location determination and its impact on intelligent transportation systems. IEEE Transactions on Intelligent Transportation Systems, 1(1), 55-64.

Zhao, Y. (2002, July). Standardisation of mobile phone positioning for 3G systems. IEEE Communications Magazine, 109-116. 